\title{
A predictive model for preterm babies born $<30$ weeks gestational age who will not attain full oral feedings
}

Daniel B. Gehle ${ }^{1}$, Alison Chapman (iD ${ }^{1}$, Mathew Gregoski (iD) ${ }^{2}$, Meghan Brunswick ${ }^{3}$, Emily Anderson ${ }^{4}$, Viswanathan Ramakrishnan ${ }^{2}$, Lutfiyya N. Muhammad ${ }^{5}$, William Head (iD) ${ }^{6}$, Aaron P. Lesher (iD ${ }^{6 凶}$ and Rita M. Ryan (iD)

(c) The Author(s), under exclusive licence to Springer Nature America, Inc. 2021

\begin{abstract}
OBJECTIVE: Develop a model to predict gastrostomy tube (GT) for feeding at discharge in infants born $<30$ weeks' (w) gestational age (GA).

STUDY DESIGN: A single-center retrospective study at academic NICU. Total of 391 (78 GT, 313 non-GT) infants < 30 w GA admitted in 2015-2018 split into test (15-16) and validation (17-18) cohorts. Classification and regression tree analysis was used to identify predictive factors for GT.

RESULTS: Several factors were associated with GT requirements. Four factors included in the model were postmenstrual age (PMA) at first oral feeding, birth GA, high-frequency ventilation exposure, necrotizing enterocolitis stage II/III. Area under the receiver operator characteristic curve was 0.944 in the test cohort, 0.815 in the validation cohort. Implementation plan based on the model was developed.

CONCLUSIONS: We developed a predictive model to risk-stratify infants born $<30 \mathrm{w}$ GA for failing full oral feeding. We hope
\end{abstract} implementation at $38 \mathrm{w}$ PMA will result in earlier placement of needed GT and discharge.

Journal of Perinatology (2022) 42:126-131; https://doi.org/10.1038/s41372-021-01219-y

\section{INTRODUCTION}

While "very preterm" ( $<32$ weeks' (w)) gestational age (GA) infants generally represent only $1.5 \%$ of all births [1] these infants experience high rates of death, medical complications, and poor neurodevelopmental outcomes, leading to significant healthcare and other associated costs [1-6]. Innovation and optimization of care to reduce morbidity, mortality, and costs associated with very and extremely preterm births is a topic of ongoing discussion [7]. One reason for prolonged hospitalizations among very preterm infants is that these patients require admission to the neonatal intensive care unit (NICU) and enteral tube feeding until they are mature enough to feed orally from the breast or bottle. While institutional protocols for feeding advancement differ significantly, the transition from naso- or oro-gastric tube to oral (PO) feeding among very premature infants is generally initiated at first sign of "readiness," which typically occurs at 32-34 weeks' postmenstrual age (PMA), when coordinated sucking, swallowing, and breathing develops [8-10]. Recent research has demonstrated that more aggressive early introduction of oral feeding at 31-32 weeks may accelerate the transition from tube to oral feeding [11-13]. However, some babies are unable to achieve full oral feeding and therefore require a nasogastric or gastrostomy tube (GT) for safe discharge. This is especially true for preterm babies with significant bronchopulmonary dysplasia, a chronic lung disease of preterm infants [13, 14]. Prolonged respiratory support, poor oral feeding advancement, together with eventual GT placement, result in prolonged hospitalizations, which likely contribute to increased costs, family inconvenience, postnatal growth deficits, and poor long-term neurodevelopmental outcomes [15-17].

In a descriptive cohort of infants at our NICU undergoing GT, pilot data from babies born 2015-2016 found that the decision to place a GT among very preterm infants may be contributing to a several week delay in discharge [18]. At our NICU, GT placement was often done after a prolonged trial of oral feeding with babies weighing far above our institutional minimum of $2.8 \mathrm{~kg}$ to undergo the surgery. Additional data from this study showed that infants born $>30 \mathrm{w}$ who required GT placement had genetic/ surgical conditions or congenital anomalies whereas infants $<30 \mathrm{w}$ required a GT due to oral feeding failure secondary to complications of prematurity [18]. This led us to use $30 \mathrm{w}$ as the threshold for the current study. Another group of investigators found that among 103 infants born at $<29$ w GA who were tube-dependent (grouped together as nasogastric, gastrostomy, or jejunostomy tubes) at discharge, $83.5 \%$ had no additional diagnoses apart from extreme prematurity [19]. These data suggest that among infants born at $<30 \mathrm{w} \mathrm{GA}$, complications of prematurity are the most common causes of oral feeding failure and thus GT requirement.

We, therefore, hypothesized that we could develop a predictive model to identify earlier which preterm infants born $<30 \mathrm{w}$

${ }^{1}$ Department of Pediatrics (Neonatology), Charleston, SC, USA. ${ }^{2}$ Department of Public Health Sciences, Charleston, SC, USA. ${ }^{3}$ Department of Pediatrics (Gastroenterology),

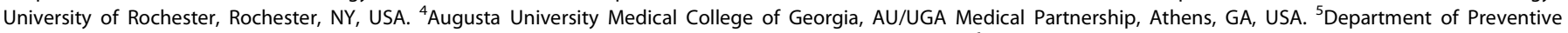

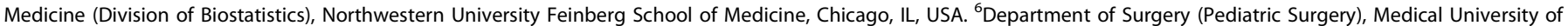
South Carolina, Charleston, SC, USA. ${ }^{7}$ Department of Pediatrics (Neonatology), Case Western Reserve University, Rainbow Babies \& Children's Hospital, Cleveland, OH, USA.

email: leshera@musc.edu

Received: 9 June 2021 Revised: 17 August 2021 Accepted: 21 September 2021

Published online: 9 October 2021 
admitted to our NICU would be at high risk of requiring GT placement for discharge. In this paper, we describe the predictive model developed for this target population and the initial plan for NICU implementation of the model.

\section{SUBJECTS AND METHODS}

This was a retrospective study with IRB approval, not requiring informed consent. This research was performed in accordance with the Declaration of Helsinki and the need for ethics committee approval was waived. All infants born $<30 \mathrm{w}$ GA admitted to the NICU at the Medical University of South Carolina in 2015-2016 were identified using a local database. Based partially on specific factors shown previously to be associated with a GT in preterm infants $[14,17,18,20-22]$ each electronic record was then examined to collect demographic data and factors that occurred during the NICU course, including gestational age (GA) at birth, birth weight (BW), race (white, black, other/unknown), sex, inborn versus outborn status, maternal illicit drug usage documented as a problem in the birth summary, neonatal abstinence syndrome documented as a problem, small for gestational age (SGA) status defined as BW $<10$ th percentile for $G A$, multiple versus singleton status, patent ductus arteriosus (PDA) considered hemodynamically significant and treated medically with indomethacin, ibuprofen or acetaminophen, PDA ligation surgery, stage II or higher necrotizing enterocolitis (NEC), NEC requiring surgery, any grade intraventricular hemorrhage (IVH), Grade III-IV IVH, ventriculomegaly, placement of a reservoir, placement of a ventriculo-peritoneal shunt, and receiving any maternal breast milk at 32 weeks' (w) post-menstrual age (PMA).

Respiratory data were collected at three time points: 30 days (d) of age, $32 \mathrm{w}$ PMA and $36 \mathrm{w}$ PMA. For each time point, the values collected were the first data point available from 0500 to 1200 on that day. We recorded $\mathrm{F}_{\mathrm{i}} \mathrm{O}_{2}$ and mode of respiratory support: none, a nasal cannula (NC), humidified high-flow NC (HFNC) used for flows $>2$ liters per minute (LPM), continuous positive airway pressure (CPAP), nasal intermittent positive pressure ventilation (NIPPV), conventional ventilation, or high-frequency ventilation (HFV) defined as either jet ventilation or an oscillator. LPM flow was recorded for babies on NC or HFNC respiratory support, while mean airway pressure (MAP) was recorded for babies on CPAP or higher positive pressure support. It was also recorded if a baby ever received HFV generally used as a "rescue" mode, at any time prior to 36 w PMA. The PMA at last ventilator day and at last CPAP day, as well as total ventilator days and total CPAP/NIPPV + ventilator days prior to $36 \mathrm{w}$ PMA were recorded to support model development.

To examine the rapidity of advancement of oral feeding, the amount of oral feeding in $\mathrm{mL} / \mathrm{kg} /$ day orally weekly from 34 to 40 w PMA, and daily for each day of oral feeding attempts once initiated from day 1 forward was recorded. Data from babies who were discharged were treated as missing rather than censored to the highest feeding volume. The PMA at which a baby was first offered oral feeding attempts was also noted.

In general, our criteria for GT placement were insufficient oral feeding volume to support adequate growth, or inability to attempt oral feeding due to underlying conditions, such as significant respiratory disease requiring tracheostomy and mechanical ventilation. Adequate growth was defined as 5-day growth velocity of $>18 \mathrm{~g} / \mathrm{kg} /$ day if weight $<1.8 \mathrm{~kg}$, $>20-25 \mathrm{~g} /$ day if weight $>1.8 \mathrm{~kg}$, or growth along a weight percentile at or above the birth weight percentile[23]. Our surgeons use a minimum weight of $2.8 \mathrm{~kg}$ as the lower threshold for laparoscopic GT placement During the study period of 2015-2018, it was our practice to allow an oral feeding trial before consideration for a GT if the infant was otherwise able to attempt oral feeding. Criteria for oral feeding attempts were respiratory support of $\leq 0.5$ LPM NC and PMA of at least $31 \mathrm{w}$. We employ occupational therapists and speech therapists to help guide with feeding safety and progression. Oral feeding advancement was determined by feeding readiness scoring and generally left to our speech team and the bedside NICU nurses who have significant expertise in this arena. Our practice was to allow for one week of exclusive breastfeeding attempts for mothers who have been pumping and planning to nurse when oral feeding is introduced. "Oral care" is provided all along with mother's milk, which provides mechanical and hormonal stimulation to the infant's oral mucosa. Oral care involves using maternal or donor milk, or sterile water, to swab the infant's oral mucosa, lips, and endotracheal tube (if applicable) every $3-6 \mathrm{~h}$ in any infant not receiving oral feeding attempts unless otherwise contraindicated. In addition, our institutional practice is to orally intubate patients, but we try to extubate them as soon as possible. Infants are not allowed to orally feed if on mechanical ventilation or CPAP but are given oral care. Infants in our NICU who receive a tracheostomy nearly always receive a GT for nutritional support, usually performed at the same time as tracheostomy. Occasionally a baby who receives a tracheostomy for an airway issue and not for primarily lung disease/ respiratory support will orally feed successfully but this is the minority.

In the initial database assessment based on aggregate data, it was noted that $14 \%$ (47 out of 329 ) of all babies $<30$ weeks required a GT for home enteral feeding (we did not use home nasogastric tube feeding). This estimate was utilized by our biostatistician team to determine a sample size of 267 patients would be adequate to achieve $90 \%$ power. Initial detailed analysis showed that babies who were discharged without GTs were discharged at a median PMA of $36.3 \mathrm{w}$, and babies who required GT underwent GT surgery at a mean of $46.0 \mathrm{w}$ PMA [18]. As a result, prior to any model development, $36 \mathrm{w}$ PMA was arbitrarily identified as the goal PMA to implement the prospective model. Exclusion criteria were developed based on a baby's ability to contribute to the model. Babies who died or were transferred to another hospital prior discharge to were excluded, as it is unknown whether they would or would not have not received a GT. We were concerned that babies admitted to our NICU after 7 days of age would make the population too heterogenous from an early feeding protocol standpoint, and some babies are transferred in to the MUSC's regional referral NICU specifically for GT placement, so they would not be appropriate to include in a predictive model based on all premature infants of a particular gestation. Thus, outborn babies admitted to MUSC after 7 days of age were excluded (Fig. 1). Other exclusions, similarly-based, are noted in our consort diagram (Fig. 1).

Babies who received a GT were compared to those who did not require a GT for discharge to home by univariate analyses using the chi-square test or Fisher's exact test for categorical variables and two-sample $t$ test or Wilcoxon rank-sum test for continuous variables. Babies who never fed orally were arbitrarily assigned a PMA at first oral feeding of $52 \mathrm{w}$ since the highest value recorded for any baby who did orally feed was $51 \mathrm{w}$. To compare oral feeding curves, linear and quadratic models were fitted to plot $\mathrm{mL} / \mathrm{kg} /$ day as a function of PMA or day of oral feeding to compare GT and non-GT babies. Babies who never had oral feeding attempts were excluded from these analyses. Classification and regression tree (CART) methodology was used to identify optimal thresholds of the predictors for determining GT requirement (yes/no) and because the final CART model produces a diagram that is easily and clinically interpretable.

The receiver operator characteristic (ROC) curve was plotted to examine the accuracy of the predictive model and the area under the curve (AUC) value was reported. The model was then internally validated on a second 2-year internal cohort of babies ( $n=187$ after exclusions) admitted to the MUSC NICU in 2017-2018, only collecting information for clinical variables included in the original predictive model, as well as selected respiratory and feeding data.

\section{RESULTS}

Data were collected retrospectively on all babies $<30 \mathrm{w}$ GA $(n=$ 281) admitted to the NICU in 2015 and 2016. Forty-seven (14\%) had GT and 234 did not. After applying exclusion criteria, full data was collected on 204 infants: 41 (20\%) with GT while 163 were discharged on full oral feeds with no GT (Fig. 1).

Many factors were significantly different between GT and nonGT babies (Table 1) on univariate analysis; factors associated with a GT at discharge were lower GA, lower BW, male sex, SGA, PDA treated medically, PDA ligation, any stage of necrotizing enterocolitis (NEC), NEC requiring surgery, any grade IVH, Grade III-IV IVH, ventriculomegaly, and HFV ever.

Every respiratory variable collected was significantly higher in the GT cohort at $30 \mathrm{~d}$ of age, $32 \mathrm{w}$ PMA and $36 \mathrm{w}$ PMA. However, since only "HFV ever" of the respiratory variables remained in the final model, detailed respiratory data will be published elsewhere to describe the typical respiratory course of infants $<30 \mathrm{w}$ GA who do or do not attain full oral feedings. Space constraints do not permit a detailed presentation here.

Linear and quadratic models were fitted to describe $\mathrm{mL} / \mathrm{kg} /$ day as a function of PMA and day of oral feeding to compare GT and non-GT babies (Fig. 2). Quadratic modeling provided a better fit for the intake by day of oral feeding data (Fig. 2A) while a linear 


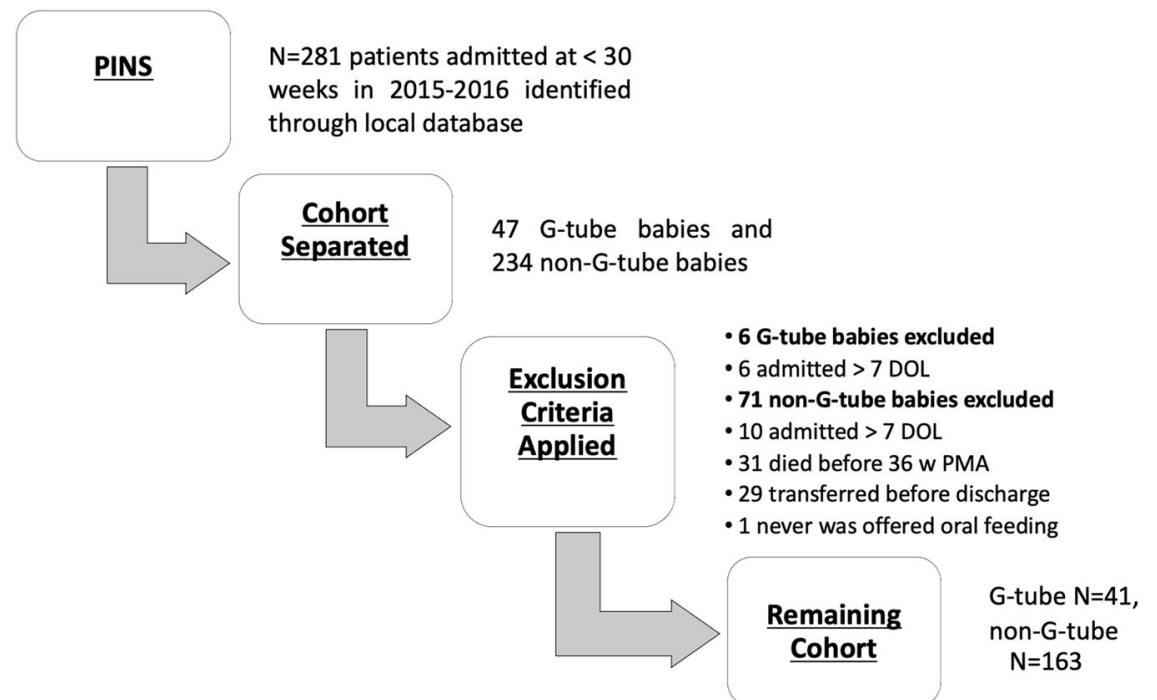

Fig. 1 Consort diagram for test cohort, demonstrating exclusion criteria application and remaining cohort. DOL day of life, PMA postmenstrual age.

Table 1. Variables and characteristics collected in the test cohort.

\begin{tabular}{|c|c|c|c|c|}
\hline & $\begin{array}{l}\text { All babies }<30 \text { weeks } \\
N=204\end{array}$ & $\begin{array}{l}\text { G-tube babies } \\
N=41\end{array}$ & $\begin{array}{l}\text { Non-G-tube babies } \\
N=163\end{array}$ & $p$-Value \\
\hline Gestational age (weeks, median (IQR)) & $27.6(26-28.6)$ & $26.3(24.3-27.4)$ & $27.9(26.4-28.9)$ & $<0.001$ \\
\hline Birth weight (grams, median (IQR)) & $977(758-1170)$ & $680(590-870)$ & $1005(833-1185)$ & $<0.001$ \\
\hline Race & & & & NS $(0.37)$ \\
\hline White & $67(33 \%)$ & $10(24 \%)$ & $57(35 \%)$ & \\
\hline Black & $119(58 \%)$ & $26(63 \%)$ & $93(57 \%)$ & \\
\hline Maternal drug use & $15(7 \%)$ & $4(10 \%)$ & $11(7 \%)$ & NS $(0.51)$ \\
\hline NAS & $4(2 \%)$ & $1(2.4 \%)$ & $3(1.9 \%)$ & NS (0.84) \\
\hline SGA & $36(18 \%)$ & $12(29 \%)$ & $24(15 \%)$ & 0.029 \\
\hline Multiple gestation & $60(30 \%)$ & $46(28 \%)$ & $14(34 \%)$ & NS $(0.60)$ \\
\hline IVH (any grade) & $62(30 \%)$ & $22(54 \%)$ & $40(25 \%)$ & $<0.001$ \\
\hline IVH Grade 3-4 & $14(7 \%)$ & $7(17 \%)$ & $7(4 \%)$ & 0.009 \\
\hline Ventriculomegaly & $9(4 \%)$ & $6(15 \%)$ & $3(2 \%)$ & 0.002 \\
\hline Reservoir & $3(1.5 \%)$ & $2(5 \%)$ & $1(0.6 \%)$ & NS $(0.10)$ \\
\hline Ventriculo-peritoneal shunt & $4(2 \%)$ & $2(5 \%)$ & $2(1 \%)$ & NS $(0.18)$ \\
\hline HFV ever & $60(29 \%)$ & $32(78 \%)$ & $28(17 \%)$ & $<0.001$ \\
\hline Any maternal milk at 32 weeks PMA & $137(67 \%)$ & $28(68 \%)$ & 109 (67\%) & NS $(0.86)$ \\
\hline PMA at first oral feeding attempt* & $34.1(32.9-36.7)$ & $40.3(36.1-52)^{*}$ & $33.6(32.6-35.6)$ & $<0.001$ \\
\hline
\end{tabular}

NAS neonatal abstinence syndrome, SGA small for gestational age, PDA patent ductus arteriosus, NEC necrotizing enterocolitis, IVH intraventricular hemorrhage, $H F V$ high-frequency ventilation, PMA post-menstrual age.

NS nonsignificant.

*Twelve babies never had any oral feeding attempts and were assigned $52 \mathrm{w}$ (highest data point was $51 \mathrm{w}$ ).

model was a better fit for the intake by PMA data (Fig. 2B). The curves were significantly different $(P<0.0001$, using a repeatedmeasures regression) in both cases, with non-GT babies exhibiting greater PO intake compared with babies who later required a GT.
The CART model from our test cohort included PMA at first oral feeding (PMAff), gestational age, HFV ever, and NEC Stage II or III ever as predictors of GT requirement. PMAff was the most predictive variable of GT requirement. The CART analysis resulted 

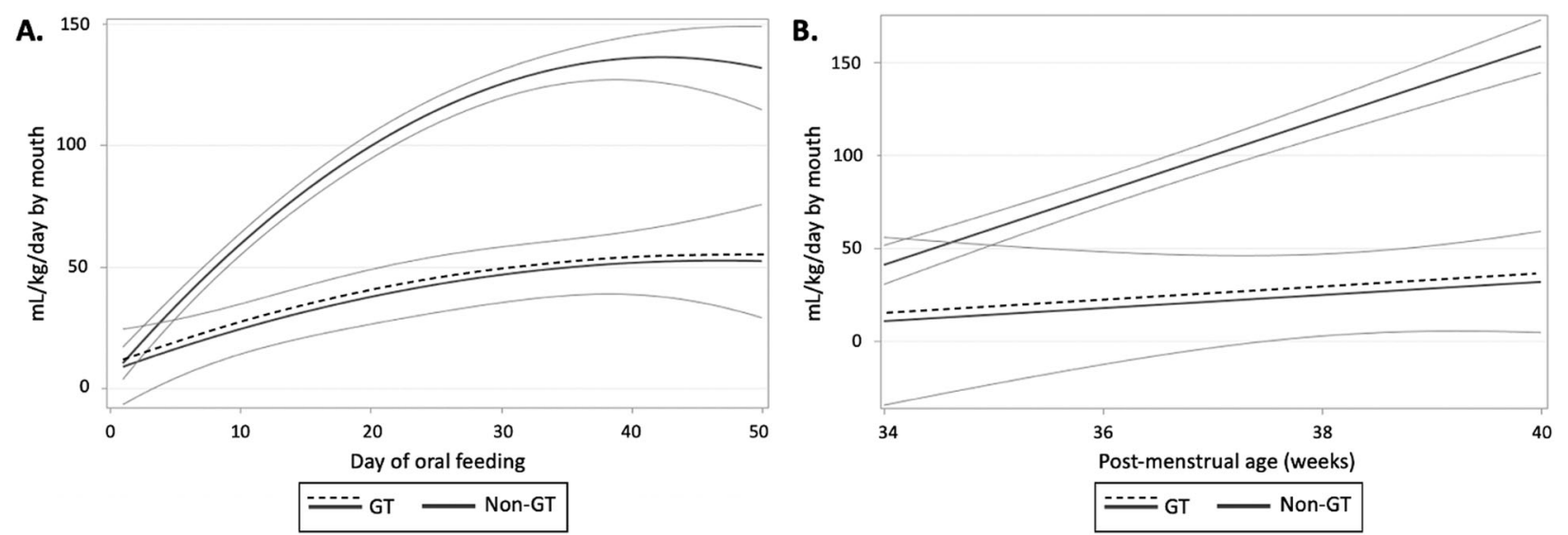

Fig. 2 Feeding data for test cohort by group. A Quadratic fit for mean $\mathrm{mL} / \mathrm{kg} /$ day by day of oral feeding attempt, with $95 \%$ confidence limits. B Linear fit for mean $\mathrm{mL} / \mathrm{kg} /$ day by PMA, with $95 \%$ confidence limits. Data from infants who never attempted oral feeding were excluded. Data from days after infants were discharged were counted as missing.

Table 2. Predictive model following CART analysis with probability for GT.

\begin{tabular}{|llllll} 
Node & Probability & $\begin{array}{l}\text { PMAff } \\
\text { (weeks) }\end{array}$ & $\begin{array}{l}\text { Gestational } \\
\text { age (weeks) }\end{array}$ & HFV ever & NEC \\
\hline 1 & 0.9999 & $\geq 46.4$ & & & \\
\hline 2 & 0.8695 & $\begin{array}{l}\geq 38 \text { and } \\
<46.4\end{array}$ & Yes & \\
\hline 3 & 0.5554 & $\geq 38$ and & No & \\
\hline 4 & 0.3447 & $<46.4$ & & Yes & Yes \\
\hline 5 & 0.49989 & $<38$ & $<24.6$ & Yes & No \\
\hline 6 & 0.36353 & $<38$ & $\geq 24.6$ & Yes & No \\
\hline 7 & 0.074 & $\begin{array}{l}\geq 35.4 \\
\text { and }<38\end{array}$ & No & \\
\hline 8 & 0.18175 & $<35.4$ & & \\
\hline
\end{tabular}

PMAff post-menstrual age at first oral feeding, HFV high-frequency ventilation, NEC necrotizing enterocolitis stage II or III.

in the generation of several "nodes" or categories of babies, which provided varying likelihoods of GT requirement based on the selected variables (Table 2, Supplementary Fig. S1). Next, a ROC curve was plotted to examine accuracy of the predictive model on the test cohort with AUC of 0.944 . A sensitivity analysis allowed us to determine that the addition of any other variable did not improve the prediction. Data were then collected for the validation cohort ( $n=187$ after applying exclusions), collecting only variables that were included in the predictive model and other selected variables (Supplementary Fig. S2). An ROC curve using the test cohort model variables demonstrated an AUC of 0.815 for the internal validation cohort (Supplementary Fig. S3). When the combined (test and validation) cohort was examined, the AUC was 0.886 .

Once the model was completed, implementation of the model in actual clinical practice was developed. We share the details of our implementation plan for anyone who wishes to utilize our concept. We used the terminal "nodes" derived directly from the CART regression analysis and examined the percent risk in each node for needing a GT (Table 2). Our model implementation plan will have the clinical team place the baby in one of the three categories based on the assessment of the four factors above at 38 w PMA; this will be done for all infants born at $<30 \mathrm{w}$ GA admitted before 7 days of age who have not achieved full oral feedings by 38 w PMA (Table 3). We generated a high-risk group (86-99\% risk of GT) with specific recommendations for expediting a GT in our institution. The medium (50-55\%) and low-medium (35\%) risk groups require close monitoring by the clinical team with daily plotting of successful oral intake on a gridded graph of days of feeding attempts versus oral intake (Supplementary Fig. S4) to help guide the clinical team in working with families to consider or not consider a GT. The low-risk group (7-18\%) needs no further intervention.

Our implementation plan does not state that any baby "must" or "should" have a G-tube; we do not intend to increase the number of GTs, but rather to have an earlier discussion so that a GT is placed sooner (with potential benefits as discussed earlier) in those infants who would have ultimately failed to attain full oral feedings. We have begun the implementation of this new protocol and will monitor for the next 2 years.

\section{DISCUSSION}

In our NICU, we were able to identify several factors predicting high risk for inability to achieve full oral feeding which in our institution leads to GT placement, and have developed a predictive model to identify future infants at high risk for oral feeding failure. The decision to place a GT can be quite difficult and significantly delays discharge to home. The purpose of this study was to provide clinicians with a tool to reasonably predict which babies would most likely fail oral feeding and require GT for discharge or to give clinicians an idea about what factors might be predictive in their own population. In this study, we propose the first model of its kind.

The predictive four variables were validated in a second cohort. Of the factors identified in the model, the most important factor was the PMAff. This is the time when the clinical team and/or unit practice would allow and encourage oral feeding. Since initiation of oral feeding attempts is mostly dependent on respiratory status, primary respiratory status variables did not remain in the model, despite high significance in univariate analysis. We are now investigating more specifically whether PMAff is actually a surrogate for respiratory status severity (Anderson et al., submitted).

The oral feeding curves may be useful to clinicians in deciding about the need for a GT. The vision for this model development was to use these data to assist in committing to a GT sooner than in our previous practice. We plan to use this tool in conjunction with the predictive model to provide prognostic information and guide decision-making conversations with parents regarding surgical tube placement. Applicability to other NICUs is uncertain and our oral feeding clinical practice guidelines (Supplementary 
Table 3. Implementation plan of the predictive model at 38 weeks PMA.

\begin{tabular}{|c|c|c|c|}
\hline $\begin{array}{l}\text { Risk of inability to } \\
\text { attain full oral feeding }\end{array}$ & $\begin{array}{l}\text { PMA at time of } \\
\text { first oral feeding }\end{array}$ & Additional factors & Next steps \\
\hline \multirow[t]{2}{*}{$\begin{array}{l}\text { High Risk } \\
(86-99 \%)\end{array}$} & $\begin{array}{l}>46.4 \text { weeks or } \\
\text { trach in place }\end{array}$ & & \multirow{2}{*}{$\begin{array}{l}\text { - Discuss with family } \\
\text { - Consult surgery } \\
\text { - Obtain upper GI study }\end{array}$} \\
\hline & 38-46.4 weeks ${ }^{3}$ & + HFV ever & \\
\hline \multirow{2}{*}{$\begin{array}{l}\text { Medium Risk } \\
(50-55 \%)\end{array}$} & 38-46.4 weeks & No HFV ever & \multirow{4}{*}{$\begin{array}{l}\text { Plot } \mathrm{mL} / \mathrm{kg} / \text { day oral feed } \\
\text { taken on feeding curve }{ }^{2}\end{array}$} \\
\hline & $<38$ weeks & $\begin{array}{l}\text { GA }<24.6 \text { weeks }+ \\
\text { HFV }\end{array}$ & \\
\hline \multirow[t]{2}{*}{$\begin{array}{l}\text { Low-Medium Risk } \\
(35 \%)\end{array}$} & $<38$ weeks & $\begin{array}{l}\mathrm{GA} \geq 24.6 \text { weeks + } \\
\mathrm{HFV}\end{array}$ & \\
\hline & $<38$ weeks & $+\mathrm{NEC}+\mathrm{HFV}$ & \\
\hline $\begin{array}{l}\text { Low Risk } \\
(7-18 \%)\end{array}$ & $<38$ weeks & No HFV ever & $\begin{array}{l}\text { Nothing further. } \\
\text { Low risk of not attaining } \\
\text { full oral feeding }{ }^{3}\end{array}$ \\
\hline \multicolumn{4}{|c|}{$\begin{array}{l}{ }^{1} \text { Model developed at MUSC with oral feeds attempted only if respiratory support } \leq 0.5 \text { LPM } \\
\text { by unit policy } \\
{ }^{2} \text { If attains } 50 \mathrm{~mL} / \mathrm{kg} / \text { day by Day } 20 \text { of oral feeding attempts, has } 95 \% \text { of successfully attainin } \\
\text { full oral feeding } \\
{ }^{3} \text { Full oral feeding ( } 140 \mathrm{~mL} / \mathrm{kg} / \text { day) } \\
\text { HFV, high-frequency ventilation (used as rescue at MUSC); NEC, necrotizing enterocolitis, } \\
\text { modified Bell's Stage II or III; GA, gestational age at birth }\end{array}$} \\
\hline
\end{tabular}

Fig. S5) are provided so others can understand the nuances that could change these data under their NICU's specific paradigms.

Babies we did not think would contribute to the model (e.g., those who died before oral feeding would be attempted) were excluded. In addition, babies admitted later in their NICU course would not have been treated the same way as our standard oral feeding preparation would be (oral care with breast milk, feeding readiness scoring, etc. see Supplementary Fig. S5) and were thus excluded.

This study is limited by the inherent heterogeneity of the underlying conditions and clinical courses of extremely preterm infants at our institution. Based on our discussion with colleagues at other centers, the process of when and whether to place a GT is quite idiosyncratic. Practice patterns in feeding, tube choice, and respiratory support management also vary widely by institution. Further, indications for GT placement in a preterm infant are numerous and include oral aversion, genetic syndromes, and cardiopulmonary, gastrointestinal, or neurological problems. Thankfully, some nuance and granularity of predicting GT requirement is clarified by our robust predictive model using only these four variables. Essentially, as we found previously [18], most babies who receive GT at our institution born $<30 \mathrm{w}$ had prematurity-related problems such as chronic lung disease or neurologic problems related to prematurity. A larger sample size would have improved our predictive power, and it is possible that addition of other variables such as illness severity scoring may have helped further refine the model. The biggest limitation is that our NICU policy of oral feeding on $\leq 0.5$ LPM flow is more restrictive than other institutions. It is possible that the delay in waiting for flow to be weaned is a time that is a "window" for neurologic development of feeding ability, and we miss this window by waiting until later to initiate oral feeding. Another limitation is that our validation cohort is internal and subject to the same limitations noted above. The endpoint for the inability to achieve full oral feeding at MUSC is a GT. GTs may be temporary and can be removed later if the baby's oral feeding improves at home $[18,22]$. Our NICU clinicians do not utilize home nasogastric tube feedings as a policy, although other NICUs do utilize this method for home feeding [24-27].

There are no consensus guidelines on the selection and timing of GT placement in preterm infants. Using our NICU population, we developed a predictive model to identify those infants born at $<30$ weeks' gestational age at highest risk for receiving GT. The four most predictive factors included in the model were PMA at first oral feed attempt, gestational age at birth, any exposure to HFV during the hospital course and NEC Stage II or III. These four factors had enough strength to predict need for GT at discharge with an AUC of 0.944 and were successfully validated on a second internal cohort. We developed and share an implementation protocol based on the model. This information may facilitate the decision to place a GT which we believe will hasten discharge. We also believe earlier discharge could theoretically improve neurodevelopmental outcomes, as well as decrease costs.

\section{REFERENCES}

1. Blencowe $H$, Cousens $S$, Chou D, Oestergaard M, Say L, Moller AB, et al. Born too soon: the global epidemiology of 15 million preterm births. Reprod Health. 2013;10(Suppl 1):S2.

2. Rogers $E E$, Hintz SR. Early neurodevelopmental outcomes of extremely preterm infants. Semin Perinatol. 2016;40(8):497-509.

3. Stoll BJ, Hansen NI, Bell EF, Shankaran S, Laptook AR, Walsh MC, et al. Neonatal outcomes of extremely preterm infants from the NICHD Neonatal Research Network. Pediatrics 2010;126(3):443-56.

4. Callaghan WM, MacDorman MF, Shapiro-Mendoza CK, Barfield WD. Explaining the recent decrease in US infant mortality rate, 2007-2013. Am J Obstet Gynecol. 2017;216(1):73.e1-e8.

5. Nassel D, Chartrand C, Doré-Bergeron MJ, Lefebvre F, Ballantyne M, Van Overmeire $B$, et al. Very preterm infants with technological dependence at home: impact on resource use and family. Neonatology 2019;115(4):363-70.

6. Institute of Medicine Committee on Understanding Premature B, Assuring Healthy O. The National Academies Collection: Reports funded by National 
Institutes of Health. In: Behrman RE, Butler AS, editors. Preterm Birth: Causes, Consequences, and Prevention. Washington (DC): National Academies Press (US) Copyright (๔ 2007, National Academy of Sciences; 2007.

7. Barfield WD. Public health implications of very preterm birth. Clin Perinatol. 2018;45(3):565-77.

8. Simpson C, Schanler RJ, Lau C. Early introduction of oral feeding in preterm infants. Pediatrics 2002;110(3):517-22.

9. Bu'Lock F, Woolridge MW, Baum JD. Development of co-ordination of sucking, swallowing and breathing: ultrasound study of term and preterm infants. Dev Med Child Neurol. 1990;32(8):669-78.

10. Mizuno K, Ueda A. The maturation and coordination of sucking, swallowing, and respiration in preterm infants. J Pediatr. 2003;142(1):36-40.

11. Kamitsuka MD, Nervik PA, Nielsen SL, Clark RH. Incidence of Nasogastric and Gastrostomy Tube at Discharge Is Reduced after Implementing an Oral Feeding Protocol in Premature (<30 weeks) Infants. Am J Perinatol. 2017;34(6):606-13.

12. Lessen BS. Effect of the premature infant oral motor intervention on feeding progression and length of stay in preterm infants. Adv Neonatal Care. 2011;11(2):129-39.

13. Grover TR, Brozanski BS, Barry J, Zaniletti I, Asselin JM, Durand DJ, et al. High surgical burden for infants with severe chronic lung disease (sCLD). J Pediatr Surg. 2014;49(8):1202-5.

14. Malkar MB, Gardner W, Welty SE, Jadcherla SR. Antecedent predictors of feeding outcomes in premature infants with protracted mechanical ventilation. J Pediatr Gastroenterol Nutr. 2015;61(5):591-5.

15. Hunt RW, Hickey LM, Burnett AC, Anderson PJ, Cheong JLY, Doyle LW. Early surgery and neurodevelopmental outcomes of children born extremely preterm. Arch Dis Child Fetal Neonatal Ed. 2018;103(3):F227-f32.

16. Hay WW Jr., Lucas A, Heird WC, Ziegler E, Levin E, Grave GD, et al. Workshop summary: nutrition of the extremely low birth weight infant. Pediatrics 1999;104 (6):1360-8.

17. Jadcherla SR, Khot T, Moore R, Malkar M, Gulati IK, Slaughter JL. Feeding methods at discharge predict long-term feeding and neurodevelopmental outcomes in preterm infants referred for gastrostomy evaluation. J Pediatr. 2017;181:125-30.e1.

18. Chapman A, George K, Selassie A, Lesher AP, Ryan RM. NICU infants who require a feeding gastrostomy for discharge. J Pediatr Surg. 2021;56(3):449-453.

19. Pahsini K, Marinschek S, Khan Z, Urlesberger B, Scheer PJ, Dunitz-Scheer M. Tube dependency as a result of prematurity. J Neonatal Perinatal Med. 2018;11(3):311-6.

20. Greene NH, Greenberg RG, O'Brien SM, Kemper AR, Miranda ML, Clark RH, et al. Variation in gastrostomy tube placement in premature infants in the United States. Am J Perinatol. 2019;36(12):1243-9.

21. Warren MG, Do B, Das A, Smith PB, Adams-Chapman I, Jadcherla S, et al. Gastrostomy tube feeding in extremely low birthweight infants: frequency, associated comorbidities, and long-term outcomes. J Pediatr. 2019;214:41-6.e5.

22. Astoria MT, Thacker L, Hendricks-Muñoz KD. Oral feeding outcome after analgesic and sedative exposure in VLBW preterm infant. Am J Perinatol. 2018;35 (14):1399-404.

23. Fenton T, Anderson D, Groh-Wargo S, Hoyos A, Ehrenkranz RA, Senterre T. An attempt to standardize the calculation of growth velocity of preterm infantsevaluation of practical bedside methods. J Pediatr. 2018;196:77-83.

24. van Kampen F, de Mol A, Korstanje J, Groof FM, van Meurs-Asseler L, Stas H, et al. Early discharge of premature infants $<37$ weeks gestational age with nasogastric tube feeding: the new standard of care? Eur J Pediatr. 2019;178(4):497-503.

25. Schuler R, Ehrhardt H, Mihatsch WA. Safety and parental satisfaction with early discharge of preterm infants on nasogastric tube feeding and outpatient clinic follow-up. Front Pediatr. 2020;8:505.
26. Williams SL, Popowics NM, Tadesse DG, Poindexter BB, Merhar SL. Tube feeding outcomes of infants in a Level IV NICU. J Perinatol. 2019;39(10):1406-10.

27. Khalil ST, Uhing MR, Duesing L, Visotcky A, Tarima S, Nghiem-Rao TH. Outcomes of infants with home tube feeding: comparing nasogastric vs gastrostomy tubes. JPEN J Parenter Enter Nutr. 2017;41(8):1380-5.

\section{AUTHOR CONTRIBUTIONS}

DBG was responsible for manuscript writing, table and figure creation, development of patient inclusion and exclusion criteria, data collection, and patient exclusion and inclusion. AC was responsible for manuscript writing, IRB approval, development of patient inclusion and exclusion criteria, data collection, and model implementation development. MG, VR, and LNM were responsible for statistical analyses and predictive model development. MB and EA were responsible for data collection. WH was responsible for data collection and paper formatting. APL was responsible for project conception and model implementation development. RMR was responsible for the project conception, development of patient inclusion and exclusion criteria, paper writing and editing, and model implementation development.

\section{COMPETING INTERESTS}

The authors declare no competing interests.

\section{ADDITIONAL INFORMATION}

Supplementary information The online version contains supplementary material available at https://doi.org/10.1038/s41372-021-01219-y.

Correspondence and requests for materials should be addressed to Aaron P. Lesher.

Reprints and permission information is available at http://www.nature.com/ reprints

Publisher's note Springer Nature remains neutral with regard to jurisdictional claims in published maps and institutional affiliations.

Attribution 4.0 International License, which permits use, sharing, adaptation, distribution and reproduction in any medium or format, as long as you give appropriate credit to the original author(s) and the source, provide a link to the Creative Commons license, and indicate if changes were made. The images or other third party material in this article are included in the article's Creative Commons license, unless indicated otherwise in a credit line to the material. If material is not included in the article's Creative Commons license and your intended use is not permitted by statutory regulation or exceeds the permitted use, you will need to obtain permission directly from the copyright holder. To view a copy of this license, visit http://creativecommons. org/licenses/by/4.0/.

(C) The Author(s), under exclusive licence to Springer Nature America, Inc. 2021 